\title{
Exact Partial Wave Expansion for an Arbitrary Optical Beams
}

\author{
A. A. R. Neves, A. Fontes, W. L. Moreira, A. A. de Thomaz, D. B. Almeida, L. C. Barbosa and C. L. \\ Cesar.
}

Instituto de Física Gleb Wataghin - Universidade Estadual de Campinas 13083-970 - Campinas - SP - Brazil.

Optical tweezers have become an important tool for biological manipulations and cell mechanical properties measurements [1]. These measurements use the displacement from equilibrium position of a microsphere as the force transducer. Therefore, the calibration procedure requires the use of good models for the optical force in microspheres. Geometrical optics has been used when the particle dimensions are much greater than the light wavelength, and Rayleigh scattering theory for the opposite. However, when the particles are of the same order of the wavelength these approximations are no longer valid. Mie resonances are typical of this size regime. Classical Mie scattering theory was developed for plane waves and cannot explain the measurements obtained using a focus beam as we have in optical tweezers. In this case, it is necessary to decompose the incident beam in plane waves relative to the center of the microsphere. As the beam focus is no longer at the origin of the coordinate system all the beam azimuthal symmetry is lost. This can be a complicated problem, especially when a full vectorial diffraction description of the electromagnetic fields and highly focused laser beams are required. All sorts of approximations and tricks have been used to proceed forward to obtain numerical results [2].

In principle this is an old subject included in text books on Electrodynamics [3] and Mathematical Physics [4], where it is common to express the fields here the partial wave expansion of incident optical beams in the form:

$$
\begin{aligned}
& \mathbf{E}_{i n c}=E_{0} \sum_{n, m}\left[\frac{i}{k} G_{n m}^{T M} \nabla \times j_{n}(k r) \mathbf{X}_{n, m}(\theta, \phi)+G_{n m}^{T E} j_{n}(k r) \mathbf{X}_{n, m}(\theta, \phi)\right] \\
& \mathbf{H}_{i n c}=\frac{E_{0}}{Z} \sum_{n, m}\left[G_{n m}^{T M} j_{n}(k r) \mathbf{X}_{n, m}(\theta, \phi)-\frac{i}{k} G_{n m}^{T E} \nabla \times j_{n}(k r) \mathbf{X}_{n, m}(\theta, \phi)\right]
\end{aligned}
$$

where $\mathbf{X}_{n, m}(\theta, \phi)$ is the vector spherical harmonic, $j_{n}(k r)$ are spherical Bessel functions and $Z=\sqrt{\mu / \varepsilon}$ is the medium impedance. The beam shape coefficients $G_{n m}^{T M}$ and $G_{n m}^{T E}$ are obtained by integrating the radial component of the fields over the solid angle

$$
j_{n}(k r)\left[\begin{array}{c}
G_{n m}^{T M} \\
G_{n m}^{T E}
\end{array}\right]=\frac{k r}{E_{0} \sqrt{n(n+1)}} \int Y_{n m}^{*}(\theta, \phi)\left[\begin{array}{c}
-E_{r} \\
Z H_{r}
\end{array}\right] d \Omega
$$

For both sides to be mathematically identical a spherical Bessel function must emerge from the solid angle integration. That is the point left in the text books, without, as far as we know, any proof that this integration over the solid angle will actually generate the spherical Bessel function necessary to cancel out the left hand side for an arbitrary optical beam. Sometimes it is suggested to use two radiuses to obtain the coefficients [3], while others propose to perform integration over the radius to get rid of the radial function on both sides [5]. 
In this report we demonstrate that for an analytical expression for the focal field instead of the traditional employed Barton-Davis beam description [6-7], the required spherical Bessel function is manifested from the solid angle integration. We will also show how it can be used to easily find an exact, closed expression, without any approximation, for the beam shape coefficients for an arbitrary beam (arbitrary location, polarization and mode) in terms of a single, for axially symmetric beams, or double integrals for non axially symmetric beams. These integrals can be numerically evaluated in a short time scale by common calculation packages which also make it very helpful for numerical evaluation. For the case of a linearly x-polarized TEM $(0,0)$ Gaussian beam the beam shape becomes,

$$
\begin{aligned}
{\left[\begin{array}{c}
G_{n m}^{T M} \\
G_{n m}^{T E}
\end{array}\right]=} & \pm 2 \pi i k f e^{-i k f} i^{n-m} e^{-i m \phi_{o}} \sqrt{\frac{2 n+1}{4 \pi n(n+1)} \frac{(n-m) !}{(n+m) !}} \int_{0}^{\alpha_{\max }} d \alpha \sqrt{\cos \alpha} e^{-f^{2} \sin ^{2} \alpha / \omega_{a}^{2}} e^{-i k z 0} \cos \alpha \\
& {\left[\left(m^{2} \frac{J_{m}\left(k \rho_{o} \sin \alpha\right)}{k \rho_{o} \sin \alpha} P_{n}^{m}(\cos \alpha)-\sin ^{2} \alpha J_{m}^{\prime}\left(k \rho_{o} \sin \alpha\right) P_{n}^{\prime m}(\cos \alpha)\right) \cos \phi_{o}\right.} \\
& \left.+i m\left(m J_{m}^{\prime}\left(k \rho_{o} \sin \alpha\right) P_{n}^{m}(\cos \alpha)-\sin ^{2} \alpha \frac{J_{m}\left(k \rho_{o} \sin \alpha\right)}{k \rho_{o} \sin \alpha} P_{n}^{\prime m}(\cos \alpha)\right) \sin \phi_{o}\right]
\end{aligned}
$$

written in terms of Bessel and associated Legendre polynomials, where $f$ is the objective focal length, $\alpha_{\max }$ is the half angle of the numerical aperture, and the center of the beam is located ate the coordinate $\left(\rho_{o}, \phi_{o}, z_{o}\right)$ from the center of the objective lens reference sphere.

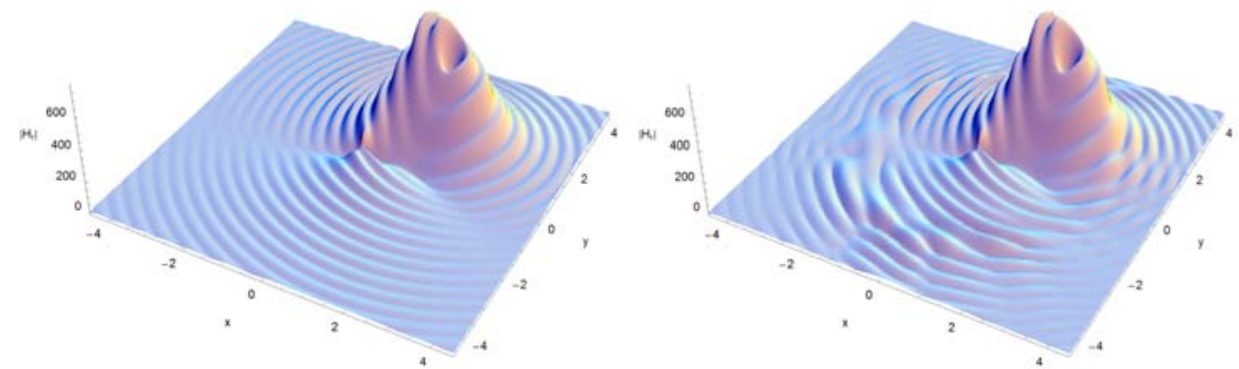

Fig.1. Absolute radial magnetic field in the $\mathrm{x}-\mathrm{y}$ plane for $785 \mathrm{~nm}$ wavelength, N.A. $=1.25$, objective filling factor of 1.8, and beam waist at $(1.5,1.5,1.5) \mu \mathrm{m}$ : (left) theoretical field; (right) Exact Partial Wave reconstruction for just the first 30 terms.

\section{References}

[1]. A. Ashkin, J. M. Dziedzic, J. E. Bjorkholm and S. Chu, Opt. Lett. 11 (1986) 288.

[2] K. F. Ren, G. Gouesbet and G. Grehan, Appl. Optics. 37 (1998) 4218.

[3] J. D. Jackson, Classical Electrodynamics, 3rd ed., John Wiley, New York, 1999.

[4] G. B. Arfken and H. J. Weber, Mathematical Methods for Physicists, 4th ed., Academic, New York, 1995.

[5] G. Gouesbet, C. Letellier, K. F. Ren and G. Grehan, Appl. Optics. 35 (1996) 1537.

[6] L. W. Davis, Phys. Rev. A. 19 (1979) 1177.

[7] J. P. Barton and D. R. Alexander, J. Appl. Phys. 66 (1989) 2800. 